\title{
A new species, Dactylosoma piperis n. sp. (Apicomplexa, Dactylosomatidae), from the pepper frog Leptodactylus labyrinthicus (Anura, Leptodactylidae) from Mato Grosso State, Brazil.
}

Letícia Pereira Úngari ${ }^{1, *}$, Edward Charles Netherlands ${ }^{2}$, André Luiz Quagliatto Santos ${ }^{3}$, Edna Paulino de Alcantara ${ }^{1}$, Enzo Emmerich ${ }^{1}$, Reinaldo José da Silva ${ }^{1}$, and Lucia Helena O’Dwyer ${ }^{1}$

${ }^{1}$ Setor de Parasitologia, DBBVPZ, Instituto de Biociências, Universidade Estadual Paulista-UNESP, Distrito de Rubião Junior, Botucatu, CEP 18.618-970, São Paulo, Brazil

${ }^{2}$ Unit for Environmental Sciences and Management, North-West University, Private Bag X6001, Potchefstroom 2520, South Africa

${ }^{3}$ Laboratório de Ensino e Pesquisa em Animais Silvestres, Faculdade de Medicina Veterinária, Universidade Federal de Uberlândia, CEP 38.400-902, Minas Gerais, Brazil

Received 28 August 2020, Accepted 25 November 2020, Published online 17 December 2020

\begin{abstract}
The Dactylosomatidae Jakowska and Negrelli, 1955 are one of four families belonging to adeleorinid coccidia and comprise the genera Babesiosoma Jakowska and Nigrelli, 1956 and Dactylosoma Labbé, 1894. These blood protozoa occur in peripheral blood of lower vertebrates, and are commonly reported parasitising amphibians. The present study describes Dactylosoma piperis n. sp. from the pepper frog Leptodactylus labyrinthicus (Spix, 1824) (Anura: Leptodactylidae), collected in 2018 at the municipality of Araguaiana, Mato Grosso State, Brazil, based on morphology of intra-erythrocytic trophozoite, primary and secondary merogonic stages and a molecular analysis (partial 18S rDNA). Dactylosoma piperis n. sp. forms a well-supported clade with other Dactylosomatidae. This is the first molecular characterization of a species of Dactylosoma from a Brazilian anuran.
\end{abstract}

Key words: Haemoparasite, Haemogregarine, Amphibian, Phylogeny, 18S rRNA.

Résumé - Une nouvelle espèce, Dactylosoma piperis n. sp. (Apicomplexa, Dactylosomatidae), parasite de la grenouille Leptodactylus labyrinthicus (Anura, Leptodactylidae) de l'état du Mato Grosso, Brésil. Les Dactylosomatidae Jakowska et Negrelli, 1955 sont l'une des quatre familles appartenant aux coccidies Adeleorina et comprennent les genres Babesiosoma Jakowska et Nigrelli, 1956 et Dactylosoma Labbé, 1894. Ces protozoaires sanguins se trouvent dans le sang périphérique des vertébrés inférieurs et sont fréquemment signalés comme parasitant des amphibiens. Ce travail décrit Dactylosoma piperis $\mathrm{n}$. $\mathrm{sp}$. de la grenouille Leptodactylus labyrinthicus (Spix, 1824) (Anura : Leptodactylidae), collectée en 2018 dans la municipalité d'Araguaiana, État du Mato Grosso, Brésil, d'après la morphologie du trophozoïte intra-érythrocytaire, des stades mérogoniques primaires et secondaires et une analyse moléculaire (ADNr $18 \mathrm{~S}$ partiel). Dactylosoma piperis $\mathrm{n}$. sp. forme un clade bien soutenu avec d'autres Dactylosomatidae. Il s'agit de la première caractérisation moléculaire d'une espèce de Dactylosoma à partir d'un anoure brésilien.

\section{Introduction}

Haemogregarines (Apicomplexa: Adeleorina) are a diverse group of blood parasites subdivided into four families: Haemogregarinidae Léger, 1911, Hepatozoidae Miller, 1908, Karyolysidae Labbé, 1984, and Dactylosomatidae Jakowska and Nigrelli, 1955. The Dactylosomatidae is a small family that currently comprises the genera Dactylosoma Labbé, 1894 and Babesiosoma Jakowska and Nigrelli, 1956 [42, 43, 50, 51].

According to Barta [1, 3], members of this family have undergone numerous reclassifications and systematic revisions,

*Corresponding author: letspungari@hotmail.com since the description of the first species, Dactylosoma ranarum (Kruse, 1890). Furthermore, there is a lack of information on the biology of this group of parasites, with the life cycles of only two species elucidated to date, namely Babesiosoma stableri Schmittner and McGhee, 1961 and Babesiosoma mariae (Hoare, 1930) [4, 6, 64]. Although leeches are considered to be the vectors of these parasites, in a recent study, possible developmental stages of Dactylosoma kermiti Netherlands, Cook and Smit, 2020 were observed in the gut and haemocoel of mosquitoes that had fed on infected hosts [64].

Species of Dactylosoma are characterised by merogonic development within the peripheral blood of their vertebrate 
hosts. During primary merogony, a large multinucleate meront is formed producing up to 16 merozoites. These merozoites then separate either repeating primary merogony or initiating secondary merogony. In secondary merogony, meronts produce up to eight merozoites that either repeat secondary merogony or mature into gamonts [65].

Currently there are six recognised species of Dactylosoma known globally. Two of these are described from fish hosts, and the remaining four species from anuran hosts. Namely D. ranarum described from the European frog Pelophylax kl. esculentus (Linnaeus, 1758); Dactylosoma sylvatica Fanthan, Porter and Richardson, 1942 reported in Lithobates sylvatica (LeConte, 1825) from Quebec, Canada; Dactylosoma taiwanensis Manwell, 1964 described infecting Fejervarya limnocharis Gravenhorst, 1829 collected in Taiwan; and D. kermiti described infecting the anurans, Ptychadena anchietae Bocage, 1868 and Sclerophrys gutturalis Power, 1927 from South Africa. Moreover, to date only two recognised species of Dactylosoma have been molecularly characterised, D. ranarum and D. kermiti, and one unidentified species of Dactylosoma from Belgium [65].

In Brazil, only two studies have reported on species of Dactylosoma from anuran hosts. Durham [23] briefly reported on two haemogregarine species infecting toads from Para State, the first species an unidentified haemogregarine possessing similar characteristics to Hemolivia stellata Petit, Landau, Baccam and Lainson, 1990 and the second a haemogregarine conforming morphologically to a species of Dactylosoma. The second was a study by Da Costa and Pereira [21] who screened a total of 100 frogs and toads captured and examined during 1964-1971 from Rio de Janeiro State, Brazil. Parasites from different groups were identified, including species of Hepatozoon and Dactylosoma. According to Da Costa and Pereira [21], a species of Dactylosoma and Hepatozoon leptodactyli were observed parasitising Leptodactylus latrans (Steffen, 1815) (syn. L. ocellatus). These authors suggested that although the dactylosomatid parasite observed resembles D. ranarum, more data are needed before final conclusions can be made. To date, there are no formal species descriptions of dactylosomatid species from Brazil.

Due to the limited data of anuran haemogregarine parasites from Brazil, the aim of this study was to characterise and describe a new species of Dactylosoma using morphological and molecular methods.

\section{Materials and methods Ethics}

All applicable international, national, and institutional guidelines for the ethical handling of animals were followed (IBAMA license 60640-1; CEUA-UNESP 1061).

\section{Anuran collection}

In August of 2018, a female adult of Leptodactylus labyrinthicus, with $105.56 \mathrm{~mm}$ snout-vent length and weight of $98 \mathrm{~g}$, was collected at the municipality of Araguaiana, Mato Grosso State, Brazil (14 $35^{\prime} 47^{\prime \prime} \mathrm{S} ; 51^{\circ} 43^{\prime} 9.59^{\prime \prime} \mathrm{W}$ ) (FAPESP
2018/09623-4; FAPESP 2018/00754-9). The animal was physically restrained and blood was collected by puncture of the cervical paravertebral sinus using sterile and disposable syringes and needles [81]. During the containment, the sex (male/female) and age of the specimen were estimated. No ectoparasites were observed on the animal.

After the blood collection, three thin blood smears were made on glass slides and the remaining blood sample was stored in EDTA tubes and frozen at $-10{ }^{\circ} \mathrm{C}$ for further molecular analysis.

\section{Morphological and morphometric analysis}

The blood smears were fixed with absolute methanol and stained with $10 \%$ Giemsa Methylene Blue Eosin Merck $^{\circledR}$ diluted in distilled water ( $\mathrm{pH} 7.0$ for $50 \mathrm{~min}$ ), according to Eisen and Schall [26], at the Parasitology division from UNESP, Botucatu. For morphological analysis of the intraerythrocytic parasite stages, digital images were captured and measured using a compound microscope at $1000 \times$ magnification with the Leica software application suite LAS V3.8 (Leica Microsystems). Measurements are in micrometres $(\mu \mathrm{m})$ comprising the parasite's length and width, with mean and standard deviation (means \pm standard deviation) given. Parasitaemia was calculated per 100 erythrocytes, with $\sim 10^{4}$ erythrocytes examined per blood smear following Cook et al. [16].

\section{Molecular analysis}

DNA was extracted from whole blood samples following the blood protocol of the DNeasy Blood and Tissue Kit (Qiagen, Valencia, CA, USA). Partial 18S rRNA gene fragments (600 bp) were amplified using the primers HepF300/Hep900 [79]. PCR amplification reactions were carried out in a final volume of $25 \mu \mathrm{L}$, containing $1 \mu \mathrm{L}$ each of 10 pmol primers, $12.5 \mu \mathrm{L}$ of Master Mix MyFi ${ }^{\mathrm{TM}}$ Mix Bioline ${ }^{\circledR}$ and $5 \mu \mathrm{L}$ of extracted DNA, with nuclease-free water accounting for the remaining volume; following the conditions of O'Dwyer et al. [69]. PCR amplification was performed on a Peltier 200 Thermocycler (MJ Research, Watertown, MA, USA), with initial denaturation at $94{ }^{\circ} \mathrm{C}$ for $3 \mathrm{~min}$, followed by 35 cycles of $94{ }^{\circ} \mathrm{C}$ for $45 \mathrm{~s}, 50{ }^{\circ} \mathrm{C}$ for $60 \mathrm{~s}$ and $72{ }^{\circ} \mathrm{C}$ for $60 \mathrm{~s}$, followed by a final extension at $72{ }^{\circ} \mathrm{C}$ for $7 \mathrm{~min}$.

PCR products were subjected to electrophoresis at $80 \mathrm{~V}$ in a $1.5 \%$ agarose gel, stained with Gel Red, and observed using ultraviolet transilluminator. The products of interest were purified by adding $2 \mu \mathrm{L}$ of ExoSAP-IT ${ }^{\circledR}$ (Affymetrix, Santa Clara, CA, USA) to $5 \mu \mathrm{L}$ of PCR product according to the manufacturer's recommendations. Amplicons were then sequenced using PCR primers on a 3500 Genetic Analyzer capillary sequencer (Applied Biosystems) and after using a BigDye Terminator Cycle Sequencing Ready Reaction Kit v.3.1 (Applied Biosystems), according to the manufacturer's recommendations.

The sequence chromatograms obtained (forward and reverse sequences) were assembled and edited using BioEdit v.7.0.9 [34] to obtain a partial $18 \mathrm{~S}$ rDNA consensus sequence. Sequences from the haemogregarine group were aligned using 
Geneious version 7.1.3 [46] with the MUSCLE algorithm (Bomatters, www.geneious.comww). Adelina dimidiata Schneider, 1875, Adelina grylli Butaeva, 1996, Klossia helicina Schneider, 1875 and Klossiella equi Baumann, 1945 from the suborder Adeleorina were selected as outgroups following Netherlands et al. [64]. Alignment gaps and ambiguities were removed using the Gblocks server [12, 76]. JModelTest v.2.1.10 [20] was used to determine the most suitable nucleotide substitution model. Based on the Akaike information criterion (AIC) the General Time Reversible [77] model with estimates of invariable sites and a discrete Gamma distribution $(\mathrm{GTR}+\mathrm{I}+\Gamma)$ was selected as the best model. Phylogenetic relationships were inferred via Bayesian inference (BI) using MRBAYES 3.2.2 [40] and Maximum likelihood (ML) analysis using RAxML 7.2.8. [32, 76], implemented in Geneious R7. For the BI analysis, the Markov Chain Monte Carlo (MCMC) algorithm was run for 1 million generations, sampling every 100 generations. The first $25 \%$ of the trees were discarded as "burn-in". The Tracer tool was used to assess convergence and the "burn-in" period [71]. For the ML analysis, nodal support was assessed using 1000 rapid bootstrap replicates [72]. The aligned sequences of Dactylosoma species from anurans were compared using a pair-wise distance (p-distance) matrix.

\section{Dactylosoma piperis Úngari, Netherlands, Silva \& O’Dwyer n. sp.}

urn:Isid:zoobank.org:act:92749FA8-8673-4556-B03FF925A15B8A07

Type-host: Leptodactylus labyrinthicus (Anura: Leptodactylidae).

Type-locality: Municipality of Araguaiana, Mato Grosso State, Brazil (coordinates $14^{\circ} 35^{\prime} 47^{\prime \prime} \mathrm{S} 51^{\circ} 43^{\prime} 9.59^{\prime \prime} \mathrm{W}$ ).

Site of infection: Peripheral blood erythrocytes.

Parasitaemia: $0.2 \%$.

Etymology: The host species L. labyrinthicus is commonly referred to in Brazil as the pepper frog. Therefore, the species epithet is derived from the Latin word piperis meaning pepper (noun in apposition).

Material deposited: Hapantotype, two blood smears from L. labyrinthicus deposited in the collection of the National Institute of Amazonian Research (INPA), Manaus, Brazil [INPA19a, INPA19b].

Gene sequence: 18S rRNA gene sequence deposited in GenBank under accession number MW264134.

Note: The authors of the new taxon are different from the authors of this paper; Article 50.1 and Recommendation 50A of the International Code of Zoological Nomenclature [41].

\section{Description (Fig. 1; Table 1):}

The developmental stages of the unidentified species of Dactylosoma observed were trophozoites, early stage meronts, meronts and merozoites from the primary merogony. For secondary merogony, it was possible to identify early stage meronts, meronts and merozoites. In addition, the early stage meronts and the mature meronts varied in morphology including the typical hand-like (dactylate shape), the quadrangular, the fan-like and circular shapes. Typically, primary merogony of species of Dactylosoma produces up to 16 merozoites and secondary merogony up to eight merozoites. However, in the present study, during primary merogony, meronts were observed producing up to ten chromatin divisions of the nuclei and during secondary merogony, meronts were observed producing up to eight chromatin divisions (Fig. 1).

\section{Primary merogony}

Trophozoite (Fig. 1A): Elongated, tapering towards one end and larger and rounded at opposite end, measuring $7.4 \mu \mathrm{m} \pm 1.3 \mu \mathrm{m}$ long, $3.75 \mu \mathrm{m} \pm 1.5 \mu \mathrm{m}$ wide, and with area of $19.31 \mu \mathrm{m}^{2} \pm 0.4 \mu \mathrm{m}^{2}$; cytoplasmic vacuoles observed mainly in tapering end; nuclei placed at the rounded end, although chromatin division is not clearly defined; cytoplasm staining whitish-purple $(n=5)$.

Young primary meronts (Fig. 1B): Ovoid to round shape with dispersed vacuoles, measuring $5.20 \mu \mathrm{m} \pm 0.15 \mu \mathrm{m}$ in length, $5.53 \mu \mathrm{m} \pm 0.7 \mu \mathrm{m}$ in width, with area of $20.41 \mu \mathrm{m}^{2} \pm 0.4 \mu^{2}$; multinucleate, with between four to six nuclei located peripherally and staining purple; causes displacement of host nuclei and cell $(n=2)$,

Primary meronts (Figs. 1C-1D): Large rounded meronts, measuring $8.59 \mu \mathrm{m} \pm 0.2 \mu \mathrm{m}$ in length, $6.73 \mu \mathrm{m} \pm 0.5 \mu \mathrm{m}$ in width, with area of $31.40 \mu \mathrm{m}^{2} \pm 0.4 \mu \mathrm{m}^{2}$; causing slight distortion and displacement of host cell nucleus; multinucleate with between 6 and 10 nuclei located peripherally; purplish or pinkish staining chromatin $(n=3)$.

Primary meronts with merozoites (Figs. 1E-1F): Large fan-shaped meronts with distinct triangular form, measuring $8.38 \mu \mathrm{m} \pm 0.1 \mu \mathrm{m}$ in length, $6.71 \mu \mathrm{m} \pm 0.25 \mu \mathrm{m}$ in width, with area of $31.24 \mu \mathrm{m}^{2} \pm 0.5 \mu \mathrm{m}^{2}(n=3)$; multinucleate with ovoid dense chromatin positioned on one side of the parasite, usually displacing erythrocyte nuclei, chromatin staining dark purple or pinkish; merozoites measurements $7.45 \mu \mathrm{m} \pm 0.25 \mu \mathrm{m}$ in length and $2.90 \mu \mathrm{m} \pm 0.25 \mu \mathrm{m}$ in width $(n=30)$.

\section{Secondary merogony}

Young secondary meront (Figs. 1G-1H): Elongated with one end tapered and the other rounded. Rounded end containing two to three nuclei, with dense and circular chromatin staining in deep magenta peripherally distributed, with or without cytoplasmic vacuole, $6.1 \mu \mathrm{m} \pm 1.2 \mu \mathrm{m}$ length, $4.15 \mu \mathrm{m} \pm 0.9 \mu \mathrm{m}$ width and $28.02 \mu \mathrm{m}^{2} \pm 0.2 \mu \mathrm{m}^{2}$ in area $(n=3)$.

Secondary meronts (Figs. 1I-1J): Dactylate (hand-like) appearance, ovoid to round shape, $6.9 \mu \mathrm{m} \pm 0.4 \mu \mathrm{m}$ length, $5.6 \mu \mathrm{m} \pm 0.2 \mu \mathrm{m}$ width and $25.53 \mu \mathrm{m}^{2}$ in area $(n=2)$; multinucleate with between five and eight nuclei located peripherally with dense chromatin staining in deep magenta.

Secondary meronts with merozoites (Figs. 1K-1L): Morphology varying from fan-like shape to quadrangular shape. Multinucleate with between six and eight nuclei with chromatin division located peripherally, with or without vacuoles; in some cases, slight displacement of host cell nucleus evident. Quadrangular shape meront (Fig. 1K): Multinucleate with six rounded nuclei, three dense nuclei positioned on each side of the meront, forming a square-shape, measuring 


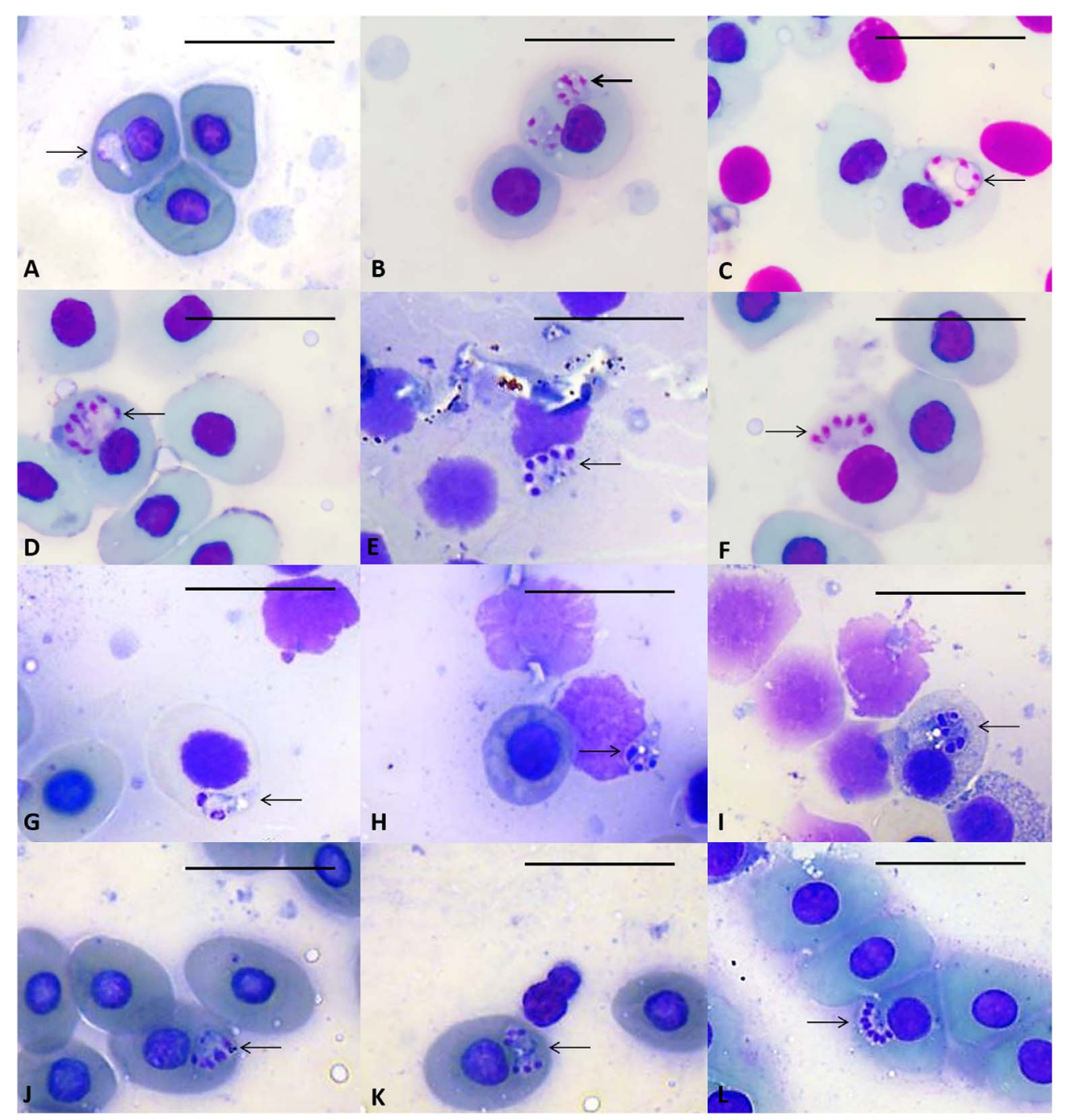

Figure 1. Dactylosoma piperis n. sp. in blood smears of Leptodactylus labyrinthicus. Primary merogony (A-F): A) Trophozoite; B) Young primary meront; C-D) Primary large rounded meronts; E-F) Fan-like shaped primary meronts with merozoites. Secondary merogony (G-L): G-H) Young secondary meronts; I-J) Secondary meronts with dactylate appearance; K-L) Secondary meronts with merozoites. Scale bar: $10 \mu \mathrm{m}$.

$7.54 \mu \mathrm{m} \pm 0.2 \mu \mathrm{m}$ long, $5.4 \mu \mathrm{m} \pm 0.9 \mu \mathrm{m}$ wide, and with area of $25.88 \mu \mathrm{m}^{2}(n=2)$. Merozoites measured $6.2 \mu \mathrm{m} \pm 0.2 \mu \mathrm{m}$ long and $1.5 \mu \mathrm{m} \pm 0.9 \mu \mathrm{m}$ wide $(n=12)$. Fan-like shape meront (Fig. 1L): Multinucleate with 8 nuclei, ovoid dense chromatin positioned on one side of meront, forming fan-like shape; usually displacing host cell nucleus, measuring $6.95 \mu \mathrm{m}$ long, $4.89 \mu \mathrm{m}$ wide, and with area of $23.68 \mu \mathrm{m}^{2}(n=1)$. Merozoites measured $5.88 \mu \mathrm{m} \pm 0.2 \mu \mathrm{m}$ long and $1.3 \mu \mathrm{m} \pm 0.9 \mu \mathrm{m}$ wide $(n=8)$.

\section{Differential diagnosis}

Dactylosoma piperis $\mathrm{n}$. sp. is characterised by its elongated and unique trophozoites, with one side rounded and the other tapered; the morphological variation of early stage meronts to mature meronts ranging between dactylate, fan-like, quadrangular and circular shapes, and the number of merozoites produced in primary merogony (up to 10) and secondary merogony (up to eight).
This species can be distinguished from all currently recognised species of Dactylosoma from anuran hosts, namely $D$. kermiti, D. ranarum, D. sylvatica, and D. taiwanensis based on several developmental characteristics, such as the number of nuclear chromatin divisions present in primary and secondary merogony, unique trophozoite morphology and developmental stage morphometrics.

In comparison, $D$. piperis $n$. sp. differs from $D$. ranarum (the first described species in the Dactylosomatidae), in the number of chromatin divisions of up to six nuclei during secondary merogony and trophozoite morphology being slender and smaller with both ends rounded. Nevertheless, certain characteristics observed, such as meronts with merozoites arranged in fan-like fashion or quadrangular mass, and the two types of schizogony (primary and secondary), are typical of dactylosomatid parasites. The first type producing larger meronts with nuclei located peripherally and vacuoles present during merozoite formation, and the second type producing smaller meronts, with chromatin division of nuclei more 
Table 1. Morphometric data on developmental stages of validated Dactylosoma species from fishes and anuran hosts around the world.

\begin{tabular}{|c|c|c|c|c|c|c|c|}
\hline \multirow[b]{2}{*}{ Species } & \multirow[b]{2}{*}{ Host(s) } & \multirow[b]{2}{*}{ Country } & \multicolumn{4}{|c|}{ Morphometric data $(\mathrm{lm})$} & \multirow[b]{2}{*}{ Reference } \\
\hline & & & $\begin{array}{c}\text { Trophozoites } \\
(\mathrm{lm})\end{array}$ & $\begin{array}{c}\text { Meronts - } \\
\text { M (lm) }\end{array}$ & $\begin{array}{l}\text { Merozoites - } \\
\text { Me }(1 \mathrm{~m})\end{array}$ & $\begin{array}{c}\text { Gametocytes } \\
(\mathrm{lm})\end{array}$ & \\
\hline $\begin{array}{l}\text { Dactylosoma } \\
\text { salvelini } \\
\text { Fantham, Porter } \\
\text { and } \\
\text { Richardson, } \\
1942\end{array}$ & $\begin{array}{l}\text { Fish } \\
\text { Salvelinus } \\
\quad \text { fontinalis } \\
\quad \text { Mitchill, } 1814\end{array}$ & Canada & N/A & $\begin{array}{c}\text { 2nd } \mathrm{M}: 5.8-8.5 \times \\
3.7-7.0\end{array}$ & N/A & $\begin{array}{c}4.4-7.8 \times \\
1.5-3.0\end{array}$ & [27] \\
\hline $\begin{array}{l}\text { Dactylosoma } \\
\quad \text { lethrinorum } \\
\text { Saunders, } 1960\end{array}$ & $\begin{array}{l}\text { Lethrinus } \\
\quad \text { nebulous } \\
\text { Forsskål, 1775; } \\
\text { L. lentjan } \\
\text { Lacepède, } 1802\end{array}$ & Egypt & N/A & 1st. M: $8.0 \times 10.5$ & $\begin{array}{c}\text { 1st. M: } \\
1.9 \times 2.4\end{array}$ & N/A & {$[73]$} \\
\hline $\begin{array}{l}\text { Dactylosoma } \\
\text { sylvatica } \\
\text { Fantham, Porter } \\
\text { and } \\
\text { Richardson, } \\
1942\end{array}$ & $\begin{array}{l}\text { Anuran } \\
\text { Lithobates } \\
\quad \text { sylvatica } \\
\quad \text { LeConte, } 1825\end{array}$ & Canada & $\begin{array}{c}\text { 1st. M: } 7.0-8.5 \times \\
\quad 6.3-7.6 \\
\text { 2nd M: } 4.4 \times 3.0\end{array}$ & $\begin{array}{c}\text { 1st. M: } 7.4-11.5 \times \\
7.0-9.3 \\
\text { 2nd M: } 5.2 \times 4.0\end{array}$ & $\begin{array}{l}\text { 2nd M: } 4.4-5.9 \times \\
1.1-2.0\end{array}$ & $\begin{array}{l}7.0-12.6 \times \\
1.5-3.0\end{array}$ & {$[27]$} \\
\hline $\begin{array}{l}\text { Dactylosoma } \\
\quad \text { taiwanensis } \\
\quad \text { Manwell, } 1964\end{array}$ & $\begin{array}{l}\text { Fejervarya } \\
\text { limnocharis } \\
\text { Gravenhorst, } \\
1829\end{array}$ & Taiwan & 1st. M: $3.9 \times 7.3$ & $\begin{array}{c}\text { 2nd. M: } 6.9-7.9 \times \\
5.6-7.3\end{array}$ & N/A & $\begin{array}{c}11.8-13.6 \times \\
2.1-2.9\end{array}$ & {$[60]$} \\
\hline $\begin{array}{l}\text { Dactylosoma } \\
\text { ranarum Kruse, } \\
1890 \text { (syn. } \\
\text { D. splendes) }\end{array}$ & $\begin{array}{l}\text { Pelophylax kl. } \\
\quad \text { esculentus } \\
\quad \text { Linnaeus, } 1758\end{array}$ & & $\begin{array}{c}\text { 1st. M: } 3.0-4.0 \times \\
1.5-2.0\end{array}$ & $\begin{array}{c}\text { 1st. M: } \\
10.0-15.0 \times \\
2.0-3.0 ; 7.3 \times 4.3 \\
\text { 2nd. M: } 9.0 \times \\
4.0 ; 4.7 \times 3.4\end{array}$ & $\begin{array}{c}\text { 1st. M: } 2.8 \times \\
0.7 ; 4.3 \times 1.3 \\
\\
\text { 2nd. M: } 2.0-3.0 \times \\
1.0-1.5 ; 3.4 \times 0.9\end{array}$ & $\begin{array}{c}5.0-8.0 \times \\
1.5-3.0 \\
7.0 \times \\
3.4\end{array}$ & {$[5,48]$} \\
\hline $\begin{array}{l}\text { Dactylosoma } \\
\text { kermiti } \\
\text { Netherlands } \\
\text { et al., } 2020\end{array}$ & $\begin{array}{l}\text { Ptychadena } \\
\quad \text { anchietae } \\
\text { Bocage, 1868; } \\
\text { Sclerophrys } \\
\text { gutturalis } \\
\text { Power, } 1927\end{array}$ & South Africa & $\begin{array}{c}\text { 1st. M: } 5.3-7.7 \times \\
2.6-4.4\end{array}$ & $\begin{array}{c}\text { 1st. M: } 8.3-12.2 \times \\
5.1-8.0 \\
\text { 2nd. M: } 5.6-8.6 \times \\
4.4-6.9\end{array}$ & $\begin{array}{c}\text { 1st. M: } 5.0-6.6 \times \\
1.8-3.2 \\
\text { 2nd. M: } 4.2-5.5 \times \\
1.8-3.5\end{array}$ & $\begin{array}{c}7.8-15.0 \times \\
1.5-3.0\end{array}$ & {$[65]$} \\
\hline
\end{tabular}

condensed and staining dark-purple with fewer merozoites produced.

For $D$. kermiti, primary merogony is characterised by up to 14 chromatin divisions and second merogony by up to six chromatin divisions, as compared to D. piperis n. sp., with up to 10 and up to 8 chromatin divisions observed in primary and secondary merogony, respectively. In addition, the trophozoites of D. kermiti are smaller and slender, and elongated to oval in shape with vacuoles present, measuring $6.7 \mu \mathrm{m} \pm 2.2 \mu \mathrm{m}$ long and $3.5 \mu \mathrm{m} \pm 1.2 \mu \mathrm{m}$ wide, which differs from the trophozoite size and shape of D. piperis n. sp. Also, the morphometric values of primary meronts and merozoites, and secondary meronts and merozoites from this study were larger as compared to D. kermiti.

In comparison, the developmental stages between D. sylvatica and D. piperis n. sp. differ in morphology and size, with trophozoites of $D$. sylvatica measuring lager (7.0-8.5 $\mu \mathrm{m} \times 6.3-7.6 \mu \mathrm{m})$, with an amoeboid shape, circular nuclei and alveolar cytoplasm without inclusions. Furthermore, meronts of $D$. sylvatica produce only up to eight merozoites, in the first and the second merogony, as compared to up to ten in the first merogony and eight in the second merogony of D. piperis $\mathrm{n}$. $\mathrm{sp}$.

With regard to D. taiwanensis and D. piperis n. sp., both species present similar trophozoite morphology, with trophozoites of D. taiwanensis measuring $3.9 \mu \mathrm{m}$ wide and $7.3 \mu \mathrm{m}$ long; distinguished morphology can be observed, with elongate or ovoid vacuolated form with equal rounded ends, compared to elongated with one end rounded and the other tapered from $D$. piperis n. sp. From secondary merogony, mature fan-like, quadrilateral-shape and hand-like meronts were observed with between four and eight nuclei, differing from the 


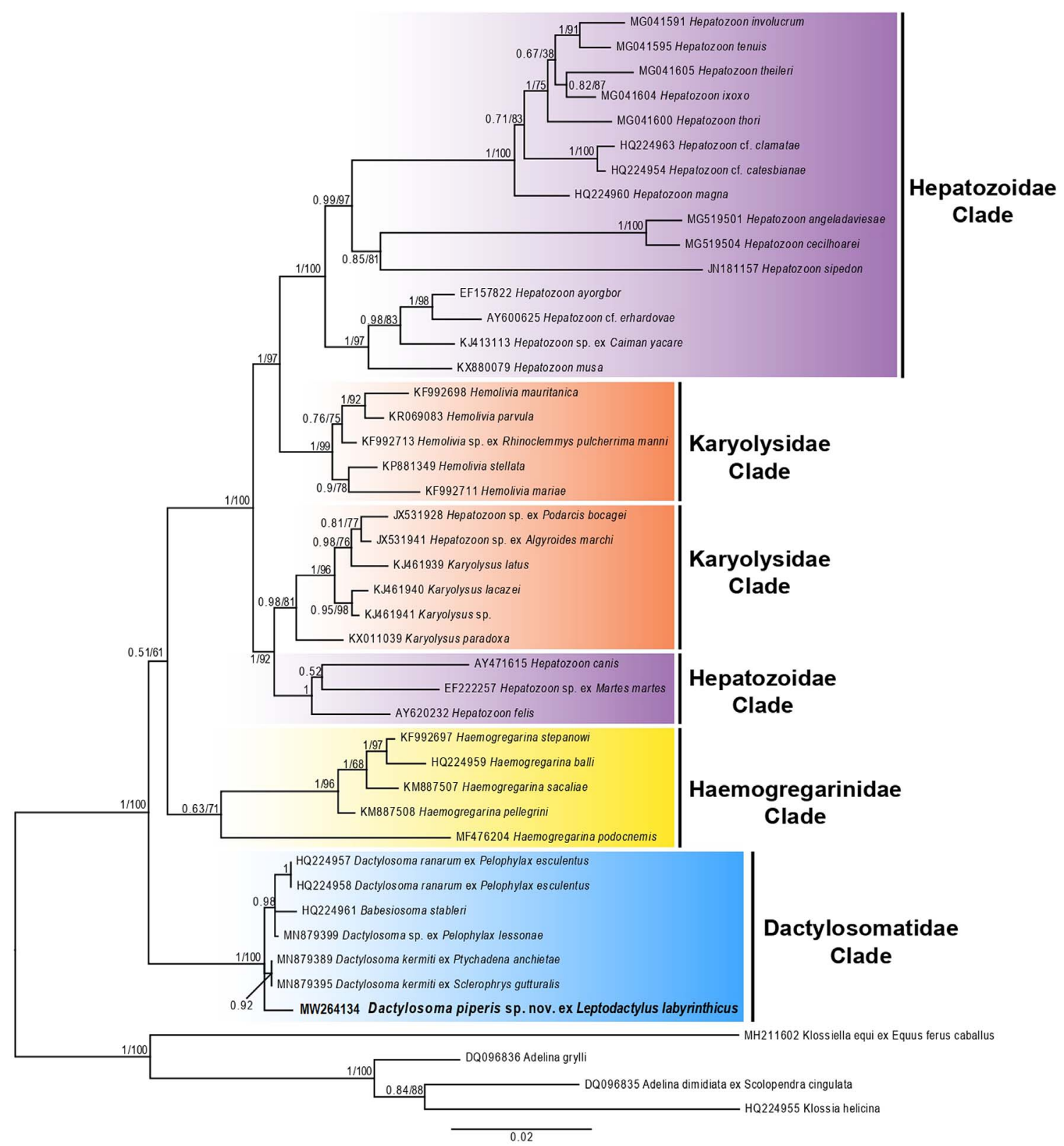

Figure 2. Consensus phylogram of haemogregarines based on 18S rDNA sequences. The topology trees with Bayesian inference (BI) and Maximum likelihood (ML) analyses were identical (represented by the ML tree). The scale bar represents 0.02 nucleotide substitutions per site. Adelina dimidiata (DQ096835), Adelina grylli (DQ096836), Klossia helicina (HQ224955) and Klossia equi (MH211602) were used as outgroups.

mature meronts of $D$. piperis n. sp. with between six and eight nuclei.

\section{Molecular and phylogenetic analysis} (Fig. 2; Tables 2-3)

The phylogenetic tree comprised sequences of adeleorinid apicomplexan parasites (Haemogregarinidae, Hepatozoidae, Karyolysidae, and Dactylosomatidae) available from GenBank (Table 2). The BI and ML phylogenetic analysis had similar topologies, showing species of Haemogregarina forming a monophyly sister to a large clade consisting of isolates from species of Hepatozoon, Hemolivia, and Karyolysus. All species of Dactylosoma clustered together as a sister group to the Haemogregarinidae clade (Fig. 2).

Dactylosoma piperis n. sp. (MW264134) is well nested within the Dactylosomatidae clade, forming a sister taxon to D. kermiti (MN879398/MN879392). Moreover, the genetic distances of the isolate from this study and dactylosomatid sequences available on GenBank showed interspecific divergence of $0.63 \%$ with D. kermiti (MN879398/MN879392) and $1.90 \%$ with D. ranarum (HQ224957/HQ224958), and the pair-wise distance varied from 0.005 to 0.009 (452 nt) (Table 3). 
Table 2. GenBank accession numbers, hosts, country and citation for SSU rDNA sequences of haemogregarines from reptiles, amphibians and mammal used in the phylogenetic analyses (except the sequence from this study).

\begin{tabular}{|c|c|c|c|c|}
\hline Species & GenBank Number & Host & Country & Citation \\
\hline $\begin{array}{l}\text { Dactylosoma kermiti Netherlands, Cook } \\
\text { and Smit, } 2020\end{array}$ & MN879392 & $\begin{array}{l}\text { Ptychadena anchietae Bocage, } \\
1868\end{array}$ & South Africa & [65] \\
\hline Dactylosoma kermiti & MN879398 & $\begin{array}{l}\text { Sclerophrys gutturalis Power, } \\
1927\end{array}$ & South Africa & {$[65]$} \\
\hline Dactylosoma ranarum Kruse, 1890 & HQ224958 & Rana esculenta Linnaeus, 1758 & Canada & {$[1]$} \\
\hline Dactylosoma ranarum & HQ224957 & Rana esculenta & Canada & {$[1]$} \\
\hline Dactylosoma sp. & MN879399 & $\begin{array}{l}\text { Pelophylax lessonae Camerano, } \\
1882\end{array}$ & Belgium & {$[65]$} \\
\hline $\begin{array}{l}\text { Babesiosoma stableri Schmittner and } \\
\text { Mc Ghee, } 1961\end{array}$ & HQ224961 & Rana septentrionalis Baird, 1854 & Canada & {$[1]$} \\
\hline $\begin{array}{l}\text { Haemogregarina podocnemis Úngari, } \\
\text { Santos, O'Dwyer, Silva, Fava, Paiva } \\
\text { and Cury, } 2018\end{array}$ & MF476204 & $\begin{array}{l}\text { Podocnemis unifilis Troschel, } \\
\quad 1848\end{array}$ & Brazil & {$[80]$} \\
\hline $\begin{array}{l}\text { Haemogregarina balli Peterson and } \\
\text { Desser, } 1976\end{array}$ & HQ224959 & $\begin{array}{l}\text { Chelydra serpentina serpentina } \\
\text { Linnaeus, } 1758\end{array}$ & Canada & {$[1]$} \\
\hline $\begin{array}{l}\text { Haemogregarina sacaliae Devořáková, } \\
2015\end{array}$ & KM887507 & $\begin{array}{l}\text { Sacalia quadriocellata } \\
\text { Siebenrock, } 1903\end{array}$ & Vietnam & [24] \\
\hline $\begin{array}{l}\text { Haemogregarina pellegrini Laveran } \\
\text { and Petit, } 1910\end{array}$ & KM887508 & $\begin{array}{l}\text { Malayemys subtrijuga (Schelegel } \\
\text { and Maller, 1845) }\end{array}$ & Vietnam & [24] \\
\hline $\begin{array}{l}\text { Haemogregarina stepanowi } \\
\text { Danilewsky, } 1885\end{array}$ & KF992697 & $\begin{array}{l}\text { Mauremys caspica (Gmelin, } \\
\text { 1774) }\end{array}$ & Turkey & {$[25]$} \\
\hline $\begin{array}{l}\text { Hemolivia stellata Petit, Landau, } \\
\text { Baccam and Lainson, } 1990\end{array}$ & KP881349 & $\begin{array}{l}\text { Amblyomma rotundatum Kock, } \\
1844\end{array}$ & Brazil & [43] \\
\hline $\begin{array}{l}\text { Hemolivia mauritanica Petit, Landau, } \\
\text { Baccam and Lainson, } 1990\end{array}$ & KF992698 & Testudo graeca Linnaeus, 1758 & Turkey & [49] \\
\hline Hemolivia parvula Dias, 1953 & KR069083 & Kinixys zombensis Hewitt, 1931 & South Africa & {$[17]$} \\
\hline $\begin{array}{l}\text { Hemolivia mariae Smallridge and } \\
\text { Paperna, } 1997\end{array}$ & KF992711 & Egernia stokesii (Gray, 1845) & Australia & [49] \\
\hline Hemolivia sp. & KF992713 & $\begin{array}{l}\text { Rhinoclemmys pulcherrima } \\
\text { manni (Dunn, 1930) }\end{array}$ & Nicaragua & [49] \\
\hline $\begin{array}{l}\text { Hepatozoon cf. catesbianae (Stebbins, } \\
\text { 1903) Desser, Hong and Martin, } \\
1995\end{array}$ & HQ224954 & $\begin{array}{l}\text { Lithobates catesbeianus (Shoaw, } \\
\text { 1802) Dubois, } 2006\end{array}$ & Canada & {$[1]$} \\
\hline $\begin{array}{l}\text { Hepatozoon ixoxo Netherlands, Cook } \\
\text { and Smit, } 2014\end{array}$ & KP119772 & $\begin{array}{l}\text { Amietophrynus maculatus } \\
\text { Hallowell, } 1854\end{array}$ & South Africa & {$[67]$} \\
\hline Hepatozoon theileri Laveran, 1905 & KJ461939 & $\begin{array}{l}\text { Amietia quecketti Boulenger, } \\
1895\end{array}$ & South Africa & {$[67]$} \\
\hline $\begin{array}{l}\text { Karyolysus paradoxa (Dias, 1954) } \\
\text { Cook, Netherlands and Smit, } 2016\end{array}$ & KX011039 & $\begin{array}{l}\text { Varanus albigularis Daudin, } \\
1802\end{array}$ & South Africa & [16] \\
\hline $\begin{array}{l}\text { Karyolysus lacazei Zechmeisterova, } \\
\text { Bellocq and Siroky, } 2019\end{array}$ & KJ461940 & Lacerta agilis Linnaeus, 1758 & Poland & [33] \\
\hline Karyolysus latus Haklová-Ko, 2014 & KJ461939 & Podarcis muralis Laurenti, 1768 & Slovakia & {$[33]$} \\
\hline Karyolysus sp. & KJ461939 & Lacerta viridis Laurenti, 1768 & Hungary & [49] \\
\hline Hepatozoon felis Patton, 1908 & AY620232 & Felis catus Linnaeus, 1758 & Spain & [14] \\
\hline Hepatozoon sp. & $\mathrm{EF} 222257$ & Martes martes Linnaeus, 1758 & Spain & {$[13]$} \\
\hline Hepatozoon canis Christophers, 1907 & AY471615 & $\begin{array}{l}\text { Pseudalopex gymnocercus } \\
\text { Fischer, } 1814\end{array}$ & Brazil & [14] \\
\hline $\begin{array}{l}\text { Hepatozoon involucrum Netherlands, } \\
\text { Cook and Smit, } 2017\end{array}$ & MG041591 & $\begin{array}{l}\text { Hyperolius marmoratus Rapp, } \\
1842\end{array}$ & South Africa & {$[66]$} \\
\hline $\begin{array}{l}\text { Hepatozoon tenuis Netherlands, Cook } \\
\text { and Smit, } 2017\end{array}$ & MG041595 & $\begin{array}{l}\text { Afrixalus fornasini (Bianconi, } \\
\text { 1849) }\end{array}$ & South Africa & {$[66]$} \\
\hline $\begin{array}{l}\text { Hepatozoon thori Netherlands, Cook } \\
\text { and Smit, } 2017\end{array}$ & MG041600 & $\begin{array}{l}\text { Hyperolius marmoratus Rapp, } \\
1842\end{array}$ & South Africa & [66] \\
\hline $\begin{array}{l}\text { Hepatozoon cf. clamatae (Stebbins, } \\
\text { 1905) Smith, } 1996\end{array}$ & HQ224963 & $\begin{array}{l}\text { Lithobates clamitans (Latreille, } \\
\text { 1801) }\end{array}$ & Canada & {$[1]$} \\
\hline $\begin{array}{l}\text { Hepatozoon magna (Grassi and Felletti, } \\
\text { 1891) Labbé, } 1899\end{array}$ & HQ224960 & $\begin{array}{l}\text { Pelophylax esculentus Linnaeus, } \\
1758\end{array}$ & Canada & [1] \\
\hline $\begin{array}{l}\text { Hepatozoon angeladaviesae Cook, } \\
\text { Netherlands, Van As and Smith, } \\
2018\end{array}$ & MG519501 & $\begin{array}{l}\text { Philothamnus hoplogaster } \\
\text { Bocage, } 1882\end{array}$ & South Africa & [13] \\
\hline
\end{tabular}


Table 2. (Continued)

\begin{tabular}{|c|c|c|c|c|}
\hline Species & GenBank Number & Host & Country & Citation \\
\hline $\begin{array}{l}\text { Hepatozoon cecilhoarei Cook, } \\
\text { Netherlands, Van As and Smith, } \\
2018\end{array}$ & MG519504 & $\begin{array}{l}\text { Philothamnus natalensis } \\
\text { natalensis (Smith, 1848) }\end{array}$ & South Africa & {$[13]$} \\
\hline $\begin{array}{l}\text { Hepatozoon sidepon Smith, Desser and } \\
\text { Martin, } 1994\end{array}$ & JN181157 & $\begin{array}{l}\text { Nerodia sipedon sipedon } \\
\quad \text { Linnaeus, } 1758\end{array}$ & Canada & {$[1]$} \\
\hline $\begin{array}{l}\text { Hepatozoon ayorgbor Sloboda, Kamler, } \\
\text { Bulantova, Votypka and Modry, } \\
2007\end{array}$ & EF157822 & Phyton regius Shaw, 1802 & Ghana & [75] \\
\hline $\begin{array}{l}\text { Hepatozoon cf. erhardovae Criado- } \\
\text { Fornelio, } 2006\end{array}$ & AY600625 & $\begin{array}{l}\text { Clethrionomys glareolus } \\
\text { Schreber, } 1780\end{array}$ & Spain & [14] \\
\hline Hepatozoon sp. & KJ413113 & Caiman yacare Daudin, 1802 & Brazil & [8] \\
\hline $\begin{array}{l}\text { Hepatozoon musa Borges-Nojosa, } \\
\text { Borges-Leite, Maia, Zanchi-Silva, } \\
\text { Braga and Harris, } 2017\end{array}$ & KX880079 & $\begin{array}{l}\text { Phylodryas nattereri } \\
\text { Steindachner, } 1870\end{array}$ & Brazil & [7] \\
\hline Hepatozoon sp. & JX531928 & $\begin{array}{l}\text { Podarcis bocagei (Lopez- } \\
\text { Seoane, 1885) }\end{array}$ & Portugal & [57] \\
\hline Hepatozoon sp. & JX531941 & $\begin{array}{l}\text { Algyroides marchi Valverde, } \\
1958\end{array}$ & Portugal & [57] \\
\hline Adelina dimidiata Schneider, 1875 & DQ096835 & $\begin{array}{l}\text { Scolopendra cingulate Latreille, } \\
1829\end{array}$ & Bulgaria & [47] \\
\hline Adelina grylli Butaeva, 1996 & DQ096836 & $\begin{array}{l}\text { Gryllus bimaculatus De Geer, } \\
1773\end{array}$ & Bulgaria & [47] \\
\hline Klossia helicina Schneider, 1875 & HQ224955 & $\begin{array}{l}\text { Cepaea nemoralis (Linnaeus, } \\
\text { 1758) }\end{array}$ & France & {$[1]$} \\
\hline Klossiella equi Baumann, 1945 & MH211602 & $\begin{array}{l}\text { Equus ferus caballus Boddaert, } \\
\quad 1785\end{array}$ & Canada & {$[56]$} \\
\hline
\end{tabular}

\section{Discussion}

Amphibians are experiencing large-scale declines in species diversity. According to the IUCN Global Amphibian Assessment over the past decade, a third of the estimated amphibian species have declined. The major contributors to amphibian's species declines are environmental changes, fragmentation, and loss of habitat [29, 30]. In addition, this group of vertebrates has a great diversity of parasites, ranging from helminths, bacteria and fungi to haemoparasites, such as trypanosomatids and haemogregarines [2, 27, 39, 54, 67, 68]. Moreover, one disease has recently caught the attention of the scientific community: the amphibian chytridiomycosis panzootic is considered the most impactful example of disease spread and demonstrates its role in the decline of amphibian biodiversity worldwide [74]. However, although parasites usually have a negative connotation, they play a fundamental role in biology, ecology, evolution and population dynamics [39].

Costa and Bérnils [19] reported that Brazil has the greatest biodiversity of amphibians in the world, with more than 1,080 described species. Yet, studies on amphibian parasites from Brazil are scarce especially with regards to protozoan haemoparasites, such as the haemogregarines [21, 23]. Therefore, there is a lack of data on the diversity, life cycles and possible vectors of protozoan haemoparasites of Brazilian anurans, highlighting the importance of screening these diverse hosts in Brazil [2, 31].

The L. labyrinthicus was infected by a species of Dactylosoma. This anuran is widely distributed throughout South America [29, 36, 37]. In Brazil, L. labyrinthicus occurs mainly near wetlands and has been recorded in open habitats throughout the Cerrado, Caatinga regions, and in central Amazonia [11, 38, 52, 57] It is a large frog from the Leptodactylus group [35] and opportunist predator feeding on invertebrate and vertebrate animals (amphibians, amphisbaenas, lizards, snakes, and small rodent species) [10, 28, 78, 81]. In the IUCN Red list, this species is classified as LC - Least Concern [35].

In the present study from the blood smears of L. labyrinthicus, a new species of Dactylosoma, Dactylosoma piperis $\mathrm{n}$. sp. is described, with parasitaemia of $0.2 \%$. In a recent study, Netherlands et al. [65] described D. kermiti infecting anurans in South Africa, with parasitaemia varying between host species and individuals. In the host Ptychadena anchietae (Bocage, 1868), parasitaemia varied from 2\% to 5.7\%, and in the host Sclerophrys gutturalis (Power, 1927), parasitaemia averaged $0.2 \%$, similar to the current study's findings.

In Brazil, studies of haemogregarine prevalence and parasitaemia from anurans are scarce. Da Sousa and Filho [22] reported $1 \%$ prevalence of Haemogregarina from 100 anurans screened. Intra-erythrocytic gamonts infecting the blood smears of one Rhinella crucifer (Wied-Neuwied, 1821) (syn. Bufo crucifer) from Rio de Janeiro State, Brazil, were found with parasitaemia $0.5 \%$.

In another study by Kattar [45], from 100 Brazilian anurans analysed, eight (8\%) were positive for haemogregarine parasites infecting blood smears of Rhinella diptycha (Cope, 1862) (syn. Bufo paracnemis) collected at João Pessoa City, Paraíba State, Brazil. However, gametocyte morphology was similar to that of the genus Hemolivia. 
Table 3. The shaded matrix (upper) shows the percentage of similarity (\%) of the nucleotide sequences and the non-shaded matrix (lower) shows the p-distance (pair-wise distance) between the Dactylosoma sequences in anurans available at GenBank (452 nt).

\begin{tabular}{lccccc}
\hline & 1 & 2 & 3 & 4 & 5 \\
\hline 1. Dactylosoma piperis n. sp. (MW264134) & & 99.27 & 99.27 & 98.91 & 98.91 \\
2. Dactylosoma kermiti (MN879398) & 0.005 & & 100 & 99.45 & 99.45 \\
3. Dactylosoma kermiti (MN879392) & 0.005 & 0.000 & & 99.45 & 99.45 \\
4. Dactylosoma sp. (MN879399) & 0.009 & 0.005 & 0.005 & 99.45 \\
5. Dactylosoma ranarum (HQ224957) & 0.009 & 0.005 & 0.005 & 0.000 & 100 \\
6. Dactylosoma ranarum (HQ224958) & 0.009 & 0.005 & 0.005 & 0.000 & 0.000 \\
\hline
\end{tabular}

Using microscopy screening of blood smears, Leal et al. [54] reported a $10 \%$ prevalence of haemogregarines in the Brazilian frogs Leptodactylus chaquensis Cei, 1950, L. podicipinus Cope, 1862 and Phyllomedusa hypocondrialis Daubin, 1800, from Mato Grosso do Sul State and São Paulo State.

Regarding species of Dactylosoma, Da Costa and Pereira [21] observed a species of Dactylosoma infecting L. latrans (Steffen, 1815) (syn. L. ocellatus) from Rio de Janeiro State with low prevalence reported only in the fall and winter season (1964-1971); however, no morphometric data are available for these observations. The only developmental stages reported were meronts conforming to secondary merogonic early meronts with nuclei located at the rounded periphery of the parasite, and a fan-like shaped meront with four nuclei.

Species of Dactylosoma have a wide distribution, infecting a variety of hosts $[9,53,60]$. These findings support the hypothesis of parasite distribution proposed by Metcalf [62], suggesting that parasite distribution could be explained by a Gondwana land link, so the same species could be reported in different hosts from distant geographic regions; however, according to Manwell [60], this theory was never accepted. However, the geographical locations of the six valid species do not include the regions of Central- and South America. Therefore, it is unlikely that $D$. piperis $\mathrm{n}$. sp., is a previously described species from a different continent, with different biomes, ecosystems and also different vertebrate hosts and possible vectors. All these data support the description of D. piperis $\mathrm{n}$. sp. as a new species with the aid of morphological and molecular analysis.

With regard to the molecular analysis, the phylogenetic relationships between different haemogregarines (Karyolysidae, Haemogregarinidae, Hepatozoidae, and Dactylosomatidae) and the isolate from the present study showed the forming of several well-supported clades. Species of Hepatozoon were polyphyletic, with species isolated from large mammals forming a well-supported clade sister to the Karyolysidae clade comprising species of Karyolysus, with species of Hepatozoon isolated from amphibians, reptiles and rodents forming a well-supported clade sister to the Karyolysidae clade comprising species of Hemolivia. The Haemogregarinidae clade formed a sister clade to the large monophyly comprising the Hepatozoidae and Karyolysidae clades. The Dactylosomatidae clade was found to be a well-supported monophyletic group sister to the Haemogregarinidae clade, these findings are similar to those reported by Netherlands et al. [65]

In addition, the Dactylosomatidae clade formed a polytomy with Babesiosoma stableri (HQ224961); Dactylosoma sp.
(MN879399) and D. ranarum (HQ224957/HQ224958) formed a monophylum; and D. kermiti (MN879398/MN879392) and D. piperis n. sp. (MW264134) nested within the polytomy. Despite the low interspecific divergence ( $p$-distance 0.0050.009 ) between dactylosomatid species, the $18 \mathrm{~S}$ gene distinguished $D$. piperis n. sp. as a separate species. Thus, although the 18S rRNA gene is a conservative marker, it has provided stability between closely related genera and species within Adeleorina [15, 18, 59, 61, 68, 70].

Moreover, in an attempt to resolve the polyphyletic genus Hepatozoon, the genus Bartazoon Karadjian, Chavatte and Landau, 2015, was proposed to replace the Hepatozoon genus from species transmitted exclusively by haematophagous insects, with the aim of resolving the polyphyletic placement of the genus Hepatozoon [44]. However, Maia et al. [58] considered the idea premature, since some issues within Hepatozoon polyphyly still remain unsolved even with the use of the proposed genus Bartazoon.

Furthermore, Léveillé et al [55], shows the problematic designation of the genus Bartazoon based on the congenic and phylogenetic relationship of Hepatozoon griseisciuri and the type species of the genus Hepatozoon as described by Miller [63]. Léveillé et al. [55] reporting complete molecular data on nuclear 18S rDNA and the mitochondrial genome from Hepatozoon spp., showed significant pairwise differences observed between $18 \mathrm{~S}$ rDNA and mitochondrial genome sequences; the sequences observed in their study support the idea of superiority of COI sequences on nuclear genes to describe species, and the mitochondrial genomes sequenced to date display staggering diversity. The adeleorinid coccidian will require additional sequence data from mitochondrial genomes to better understand the taxonomy and phylogenetic classifications, and the authors suggested that the genus Hepatozoon is likely distributed into multiple genera that have yet to be defined.

Notwithstanding, until the scientific community has complete knowledge about the transmission structure and life cycle of haemogregarines, as well as the real phylogenetic and genomic diversity, the addition of a new genus to the group is precipitated. Thus, the phylogenetic analysis from this study was based on Léveillé et al. [55] and Maia et al. [58], within the old classification of Hepatozoon spp., covering different groups of animals and transmission pathways, considered valid so far.

The importance of using techniques to correctly identify and describe a new species is emphasized in this manuscript. However, regarding the molecular technique, future studies should include using variable markers, such as a mitochondrial 
gene, to increase the phylogenetic resolution and systematic position on dactylosomatid parasites. Furthermore, studies including a great variety of Brazilian anuran species from different localities should be done with the aim of increasing the biodiversity and prevalence knowledge of dactylosomatid species. Also, studies focusing on life-cycle experimental work, testing possible vectors in the transmission D. piperis $\mathrm{n}$. sp., should be attempted to gain a better understanding of the ecology of this parasite.

This study provides the first report with molecular characterisation of a species of Dactylosoma parasitising Brazilian anurans.

\section{Conflict of interest}

The authors declare that they have no conflict of interest.

Acknowledgements. We thank the team of the Laboratory for Teaching and Research in Wild Animals (LAPAS) and a nongovernmental organisation for the preservation of wild animals in Brazil (ONG PAS).

\section{Funding}

R.J.S. is supported by FAPESP (2016/50377-1), CNPq (309125/2017-0), and CNPq-PROTAX (440496/2015-2). L.P. $\mathrm{U}$ is supported by FAPESP (2018/00754-9; 2018/09623-4). L.H.O is supported by FAPESP (2018/09623-4).

\section{References}

1. Barta JR, Ogedengbe JD, Martin DS, Smith TG. 2012. Phylogenetic position of the adeleorinid coccidia (Myzozoa, Apicomplexa, Coccidia, Eucoccidiorida, Adeleorina) inferred using $18 \mathrm{~S}$ rDNA sequences. Journal of Eukaryotic Microbiology, 59(2), 171-180.

2. Barta JR. 2000. Adeleorina. In: Lee JJ, Leedale GF, Bradbury P. An Illustrated Guide to the Protozoa, 2nd ed. Allen Press Inc: KS, USA. pp. 305-318.

3. Barta JR. 1991. The Dactylosomatidae. Advances in Parasitology, 30, 1-37.

4. Barta JR, Boulard Y, Desser SS. 1987. Ultrastructural observations on secondary merogony and gametogony of Dactylosoma ranarum Labbe, 1894 (Eucoccidiida: Apicomplexa). Journal of Parasitology, 73, 1019-1029.

5. Barta JR, Desser SS. 1986. Light and electron microscopic observations on the intraerythrocytic development of Babesiosoma stableri (Apicomplexa, Dactylosomatidae) in frogs from Algonquin Park, Ontario. Journal of Protozoology, 33, 359-368.

6. Barta JR, Desser SS. 1989. Development of Babesiosoma stableri (Dactylosomatidae; Adeleina; Apicomplexa) in its leech vector (Batracobdella picta) and the relationship of the dactylosomatids to the piroplasms of higher vertebrates. Journal of Protozoology, 36, 241-253.

7. Borges-Nojosa DM, Borges-Leite MJ, Maia JP, Zanchi-Silva D, da Rocha BR, Harris DJ. 2017. A new species of Hepatozoon Miller, 1908 (Apicomplexa: Adelerina) from the snake Philodryas nattereri Steindachner (Squamata: Dipsadidae) in northeastern Brazil. Systematic Parasitology, 94, 65-72.
8. Bouer A, André LR, Luzzi MC, Oliveira JP, Rodrigues AC, Varani AM, Miranda VFO, Perles L, Wherther K, Machado RZ. 2017. Hepatozoon caimani in Caiman crocodilus yacare (Crocodylia, Alligatoridae) from North Pantanal, Brazil. Revista Brasileira de Parasitologia Veterinária, 26, 352-358.

9. Boulard Y, Vivier E, Landau I. 1982. Ultrastructure de Dactylosoma ranarum (Kruse, 1890); affinités avec les coccidies; révision du statut taxonomique des dactylosomides. Protistologica, 18, 103-112.

10. Cardoso AJ, Sazima I. 1977. Batracofagia na fase adulta e larvária da rã-pimenta, Leptodactylus labyrinthicus (Spix 1824 Anura, Leptodactylidae. Ciência e Cultura, 29(10), 1130-1132.

11. Carvalho AL, Nelson BW, Bianchini MC, Plagnol D, Kuplich TM, Daly DC. 2013. Bamboo-dominated forests of the Southwest Amazon: detection, spatial extent, life cycle length and flowering waves. PlosOne, 8(1), e54852.

12. Castresana J. 2000. Selection conserved blocks from multiple alignments for their use in phylogenetic analysis. Molecular Biology and Evolution, 17, 540-552.

13. Criado Fornelio A, Buling A, Casado N, Gimenez C, Ruas J, Wendt L, da Rosa-Farias N, Pinheiro M, Rey-Valerion C, Barba-Carretero JC. 2009. Molecular characterization of arthropod-borne hematozoans in wild mammals from Brazil, Venezuela and Spain. Acta Parasitologica, 54(3), 187-193.

14. Criado-Fornelio A, Ruas JL, Casado N, Farias NAR, Soares MP, Müller G, Brum JCW, Berne MEA, Buling-Saraña A, Barba-Carretero JC. 2006. New molecular data on mammalian Hepatozoon species (Apicomplexa: Adeleorina) from Brazil and Spain. Journal of Parasitology, 92(1), 93-99.

15. Cook CA, Netherlands EC, Van As J, Smit NJ. 2018. Two new species of Hepatozoon (Apicomplexa: Hepatozoidae) parasiting species of Philothamus (Ophidia: Colubridae) from South Africa. Folia Parasitologica, 65, 2-11.

16. Cook CA, Netherlands EC, Smith NJ. 2016. Redescription, molecular characterization and taxonomic re-evaluation of a unique African monitor lizard haemogregarine Karyolysus paradoxa (Dias, 1954) n. comb. (Karyolysidae). Parasites \& Vectors, 9(1), 347-359.

17. Cook CA, Netherlands EC, Smit NJ. 2015. First Hemolivia from Southern Africa: Reassigning chelonian Haemogregarina parvula Dias, 1953 (Adeleorina: Haemogregarinidae) to Hemolivia (Adeleorina: Karyolysidae). African Zoology, 50(2), 165-173.

18. Cook CA, Smit NJ, Davies AJ. 2009. A redescription of Haemogregarina fitzsimonsi Dias, 1953 and some comments on Haemogregarina parvula Dias, 1953 (Adeleorina: Haemogregarinidae) from Southern African tortoises (Cyptodira: Testudinidae) with new host data and distribution records. Folia Parasitologica, 56, 173-179.

19. Costa H, Bérnils RS. 2018. Répteis do Brasil e suas Unidades Federativas: lista de espécies. Herpetologia Brasileira, 7, 11-57.

20. Dariba D, Toboada GL, Doallo R, Posada D. 2012. JModelTest 2: more models, new heuristics and parallel computing. Nature Methods, 9, 772.

21. Da Costa SCG, Pereira NM. 1971. Lankesterella alencari n. sp., a Toxoplasma-like organism in the Central Nervous System of Amphibia (Protozoa, Sporozoa). Memórias do Instituto Oswaldo Cruz, 69(3), 397-411.

22. De Sousa MA, Filho AB. 1974. Uma nova hemogregarina no sangue de Bufo crucifer Wied, 1821 do Brasil. Memórias do Instituto Oswaldo Cruz, 72(3/4), 275-282.

23. Durham HE. 1902. Report on the yellow fever expedition to Para of the Liverpool School of Tropical Medicine and Medical Parasitology. Longmans, Breen and Co.: London. 
24. Dvořaková N, Kvičerová J, Hostovský M, Siroký P. 2015. Haemogregarines of freshwater turtles from Southeast Asia with a description of Haemogregarina sacaliae sp. $\mathrm{n}$. and a redescription of Haemogregarina pellegrini Laveran and Pettit, 1910. Parasitology, 142(6), 816-826.

25. Dvořaková N, Kvičerová J, Papoušek I, Javanbakht H, Tiar G, Kami H, Siroký P. 2014. Haemogregarines from western Palaearctic freshwater turtles (genera Emys, Mauremys) are conspecific with Haemogregarina stepanowi Danilewsky, 1885. Parasitology, 141(4), 522-530.

26. Eisen RJ, Schall JJ. 2000. Life history of a malaria parasite (Plasmodium mexicanum): independent traits and basis for variation. Proceedings. Biological Sciences, 267, 793-799.

27. Fantham HB, Porter A, Richardson LR. 1942. Some haematozoa observed in vertebrates in Eastern Canada. Parasitology, 34, 199-226.

28. Fonseca E, Lanna F, Carvalho R, Gehara M. 2012. Predation on Sibynomorphus neuwiedi (Serpentes: Dipsadidae) by Leptodactylus labyrinthicus (Anura: Leptodactylidae) in southeastern Brazil. Herpetology Notes, 5, 167-168.

29. Frost DR. 2019. Amphibian Species of the World. American Museum of Natural History: New York, USA. Accessible at: http://research.amnh.org/herpetology/amphibia/index.html

30. Gibbons JW, Scott DE, Ryan TJ, Buhlmann KA, Tuberville TD, Metts BS, Greene JL, Mills T, Leiden Y, Poppy S, Winne CT. 2000. The global decline of reptiles, déjà vu amphibians. BioScience, 50, 553-556.

31. Godfrey RD Jr, Fedynich AM, Pence DB. 1987. Quantification of haemotozoa in blood smears. Journal of Wildlife Diseases, $23,558-565$.

32. Guindon S, Gascuel O. 2003. A simple, fast, and accurate algorithm to estimate large phylogenies by maximum likelihood. Systematic Biology, 52, 696-704.

33. Haklová-Kočiková B, Hižňanová A, Majláth I, Račka K, Harris DJ, Földvári G, Tryjanowski P, Kokošová N, Malčeková B, Kajláthová V. 2014. Morphological and molecular characterization of Karyolysus neglected but common parasite infecting some European lizards. Parasites \& Vectors, 10(7), 555-566.

34. Hall T. 2011. BioEdit: an important software for molecular biology. GERF Bulletin of Biosciences, 2, 60-61.

35. Heyer R, Mijares A, Baldo D. 2008. Leptodactylus labyrinthicus. The IUCN Red Listo f Threatened Species, 2008, 2020. https:// dx.doi.org/10.2305/IUCN.UK.2008.RLTS.T57137A11589949.en. Downloaded 17 April.

36. Heyer WR. 2005. Variation and taxonomic clarification of the large species of the Leptodactylus pentadactylus species group (Amphibia: Leptodactylidae) from middle America, Northern South America, and Amazonia. Arquivos de Zoologia, 37(3), 269-348.

37. Heyer WR. 1995. South American rocky habitat Leptodactylus (Amphibia: Anura: Leptodactylidae) with descrition of two new species. Proceedings of the Biological Society of Washington, 108, 695-716.

38. Heyer WR. 1979. Systematics of the pentadactylus species group of the frog genus Leptodactylus (Amphibia: Leptodactylidae). Smithsonian Contributions to Zoology, 301, 1-43.

39. Holmes JC, Price P. 1986. Communities of parasites 187-213, in Community ecology: patterns and processes. Anderson DJ, Kikkawa J, Editors. Blackwell Scientific Publications: Oxford.

40. Huelsenbeck JP, Ronquist F. 2001. MRBAYES: Bayesian inference of phylogenetic trees. Bioinformatics, 17, 754-755.

41. ICZN. 1999. International Code of Zoological Nomenclature. The International Trust for Zoological Nomenclature: London. http://www.nhm.ac.uk/hosted-sites/iczn/code/.
42. Jakowska S, Nigrelli RF. 1955. A taxonomic re-evaluation of Dactylosoma Labbé. 1894, a babesioid of cold-blooded vertebrates. Journal of Protozoology, 2, 8.

43. Jakowska S, Nigrelli RF. 1956. Babesiosoma gen. nov. and other babesioids in erythrocytes of cold-blooded vertebrates. Annals of the New York Academy of Sciences, 64, 112-127.

44. Karadjian G, Chavatte JM, Landau I. 2015. Systematic revision of the adeleid haemogregarines, with creation of Bartazoon n. g., reassignment of Hepatozoon argantis Garnham, 1954 to Hemolivia, and molecular data on Hemolivia stellata. Parasite, 22, 31.

45. Kattar MR. 1986. Ocorrência de uma haemogregarina (Protozoa, Apicomplexa) em Bufo paracnemis Lutz, 1925. Boletim de Zoologia, 10, 189-196.

46. Kearse M, Moir R, Wilson A, Stones-Havas S, Cheung M, Sturrock S, Buxton S, Cooper A, Markowitz S, Duran C, Thierer T, Ashton B, Meintjes P, Drummond A. 2012. Geneious Basic: An integrated and extendable desktop software platform for the organization and analysis of sequence data. Bioinformatics, 28, 1647-1649.

47. Kopečná J, Jirků M, Oborník M, Tokarev YS, Lukeš J, Modrý D. 2006. Phylogenetic analysis of coccidian parasites from invertebrates: Search from missing links. Protist, 157(2), 173-183.

48. Kruse W. 1890. Archiv für pathologische Anatomie und Physiologie und für klinische Medicin, 120, 541-560.

49. Kvičerová J, Hypša V, Dvořaková N, Mikulíček P, Jandzik D, Gardner MG, Javanbakht H, Siroký P. 2014. Hemolivia and haemogregarines with tangled evolutionary relationships. Protist, 165(5), 688-700.

50. Labbé A. 1894. Recherches zoologiques et biologiques sur les parasites endoglobulaires du sang des vertébrés. Archives de Zoologie Expérimentale et Générale, 2, 255-259.

51. Lankester ER. 1871. On Undulina, the type of a new group of Infusoia. Journal of Cell Science, 11, 387-389.

52. Larson PM, De Sá RO. 1998. Chondrocranial morphology of Leptodactylus larvae (Leptodactylidae: Leptodactylinae): its utility in phylogenetic reconstruction. Journal of Morphology, 238, 287-305.

53. Leal DDM, Dreyer CS, Da Silva RJ, Ribolla PEM, Paduan KS, Blanchi I, O'Dwyer LH. 2015. Characterization of Hepatozoon spp. in Leptodactylus chaquensis and Leptodactylus podicipinus from two regions of the Pantanal, state of Mato Grosso do Sul, Brazil. Parasitology Research, 114, 1541-1549.

54. Leal DDM, O'Dwyer LH, Ribeiro VC, Silva RJ, Ferreira VL, Rodrigues RB. 2009. Hemoparasites of the genus Trypanosoma (Kitenoplastida: Trypanosomatidae) and hemogregarines in Anurans of the São Paulo and Mato Grosso do Sul States - Brazil. Anais da Academia Brasileira de Ciências, 81(2), 199-206.

55. Léveillé AN, El Skhawy N, Barta JR. 2020. Multilocus sequencing of Hepatozoon cf. griseisciuri infections in Ontario eastern gray squirrels (Sciurus carolinensis) uncovers two genotypically distinct sympatric parasite species. Parasitology Research, 119, 713-724.

56. Léveillé AN, Bland SK, Carlton K, Larouche CB, Kenney DG, Brouwer ER, Lillie BN, Barta JR. 2019. Klossiela equi infecting kidneys of Ontario Horses: Life cycle features and multilocus sequence-based genotyping confirm the genus Klossiella belongs in the Adeleorina (Apicomplexa: Coccidia). Journal of Parasitology, 105(1), 29-40.

57. Lima AP, Magnusson WE, Menin M, Erdtmann LK, Rodrigues DJ, Keller C, Hödl W. 2008. Guia de sapos da Reserva Adolpho Ducke, Amazônia central (Guide to the frogs of Reserva Adolpho Ducke, central Amazonia). Áttema: Manaus. p. 168.

58. Maia JPMC, Carranza S, Harris DJ. 2016. Comments on the systematic revision of adeleid haemogregarines: are more data needed? Journal of Parasitology, 102, 549-552. 
59. Maia JPMC, Perera A, Harris DJ. 2012. Molecular survey and microscopic examination of Hepatozoon Miller, 1908 (Apicomplexa: Adeleorina) in lizards from western Mediterranean. Folia Parasitologica, 59(4), 241-248.

60. Manwell RD. 1964. The genus Dactylosoma. Journal of Protozoology, 11, 526-530.

61. Matthew JS, Van Den Bussche RA, Ewing SA, Malayer JR, Latha BR, Panciera RJ. 2000. Phylogenetic relationships of Hepatozoon (Apicomplexa: Adeleorina) based on molecular, morphologic and life-cycle characters. Journal of Parasitology, 86(2), 366-372.

62. Metcalf MM. 1929. The Opalinidae and their significance. Proceeding of the National Academy of Sciences of the United State of America, 15, 448-452.

63. Miller WW. 1908. Hepatozoon perniciosum (n.g., n.sp.): a haemogregarine pathogenic for white rats, with a description of the sexual cycle in the intermediate host, a mite (Lelaps echidninus). Government Printing Office: Washington.

64. Negm-Eldin MM. 1998. Life cycle, host restriction and longevity of Babesiosoma mariae Hoare, 1930 (Apicomplexa: Dactylosomatidae). Deutsche tierarztliche Wochenschrift, 105, 367-374.

65. Netherlands EC, Cook CA, Du Preez LH, Vanhove MPM, Brendonck L, Smit NJ. 2020. An overview of the Dactylosomatidae (Apicomplexa: Adeleorina: Dactylosomatidae), with the description of Dactylosoma kermiti n. sp. parasiting Ptychadena anchietae and Sclerophrys guttaralis from South Africa. International Journal of Parasitology: Parasites and Wildlife, 11, 246-260.

66. Netherlands EC, Cook CA, Du Preez LH, Vanhove MPM, Brendonch L, Smit NJ. 2018. Monophyly of the species of Hepatozoon (Adeleorina: Hepatozoidae) parasiting (African) anurans, with the description of three new species from hyperoliid frogs in South Africa. Parasitology, 145, 1039-1050.

67. Netherlands EC, Cook CA, Smit NJ. 2014. Hepatozoon species (Adeleorina: Hepatozoidae) of African bufonids, with morphological description and molecular diagnosis of Hepatozoon ixoxo sp. nov. parasiting three Amietophrynus species (Anura: Bufonidae). Parasites \& Vectors, 7, 552.

68. O'Donoghue P.. 2017. Haemoprotozoa: making biological science of molecular phylogenies. International Journal of Parasitology: Parasites and Wild-Life Diseases, 6, 241-256.

69. O'Dwyer LH, Moço TC, Paduan Kdos S, Spenassatto C, da Silva RJ, Ribolla PE. 2013. Description of three new species of Hepatozoon (Apicomplexa, Hepatozoidae) from Rattlesnakes (Crotalus durissus terrificus) based on molecular, morphometric and morphologic characters. Experimental Parasitology, 135(2), 200-207.

70. Oyamada M, Davoust B, Boni M, Dereure J, Buchetion B, Hammad A, Itamoto K, Okuda M, Inokuma H. 2005. Detection of Babesia canis rossi, B. canis vogeli, and Hepatozoon canis in dogs in a village of eastern Sudan by using a screening PCR and screening methodologies. Clinical and Diagnostic Laboratory Immunology, 12(11), 1343-1346.

71. Rambaut A, Drummond AJ, Xie D, Baele G, Suchard MA. 2018. Posterior summarisation in Bayesian phylogenetics using Tracer 1.7. Systematic Biology, 67, 901-904.

72. Rambaut A. 2012. FigTree v1.4. Molecular evolution, phylogenetics and epidemiology.

73. Saunders DC. 1960. A survey of the blood parasites in the fishes of the Red Sea. Transactions of the American Microscopical Society, 79, 239-252.

74. Scheele BC, Pasmans F, Skerratt LF, Berger L, Martel A, Beukema W, Acevedo AA, Burrowes PA, Carvalho T, Catenezzi A, De La Riva I, Fisher MC, Flechaqs SV, Foster CN, Frías-Álvarez P, Garner TWJ, Gratwicke B, Guyasamin JM, Hirschfeld M, Kolby JE, Kosch TA, La Marca E, Lindenmayer DB, Lips KR, Longo AV, Maneyro R, McDonald CA, Mendelson J 3rd, Palacios-Rodriguez P, Parra-Olea G, Richards-Zawachi CL, Rödel M, Rovito SM, Soto-Azat C, Toledo LP, Voyles J, Weldon C, Whitfield SM, Wilkinson M, Zamudio KR, Canessa S. 2019. Amphibian fungal panzootic causes catastrophic and ongoing loss of biodiversity. Science, 363(6434), 1459-1463.

75. Silva WR, Giaretta AA. 2008. Further notes on the natural history of the South American pepper frog, Leptodactylus labyrinthicus (Spix 1824) (Anura, Leptodactylidae). Brazilian Journal of Biology, 68(2), 403-407.

76. Stamatakis A. 2014. RAxML version 8: a tool for phylogenetic analysis and post-analysis of large phylogenies. Bioinformatics, 30, 1312-1313.

77. Tavaré S. 1986. Some probabilistic and statistical problems in the analysis of DNA sequences. Lectures on Mathematics in the Life Sciences, 17, 57-86.

78. Toledo LF, Ribeiro RS, Haddad CFB. 2007. Anurans as prey: an exploratory analysis and size relationships between predators and their prey. Journal of Zoology, 271, 170-177.

79. Ujvari B, Marques EJ. 2005. High prevalence of Hepatozoon spp. (Apicomplexa: Hepatozoidae) infection in water pythons (Liasis fuscus) from tropical Australia. Journal of Parasitology, 90, 670-672.

80. Úngari LP, Santos ALQ, Odwyer LH, Silva MRL, Fava NNM, Paiva GCM, Pinto RMC, Cury MC. 1885. (Adeleina: Haemogregarinidae) in free-living and captive yellow-spotted river turtles Podocnemis unifilis (Testudines: Podocnemidae) from Brazil. Parasitology Research, 117(5), 1535-1548.

81. Vaz-Silva W, Silva HLR, Silva NJ Jr. 2003. Leptodactylus labyrinthicus (Labyrinth Frog). Diet. Herpetological Review, $34,359$.

Cite this article as: Úngari LP, Netherlands EC, Quagliatto Santos AL, de Alcantara EP, Emmerich E, da Silva RJ \& O’Dwyer LH. 2020. A new species, Dactylosoma piperis n. sp. (Apicomplexa, Dactylosomatidae), from the pepper frog Leptodactylus labyrinthicus (Anura, Leptodactylidae) from Mato Grosso State, Brazil. Parasite 27, 73. 
Reviews, articles and short notes may be submitted. Fields include, but are not limited to: general, medical and veterinary parasitology; morphology, including ultrastructure; parasite systematics, including entomology, acarology, helminthology and protistology, and molecular analyses; molecular biology and biochemistry; immunology of parasitic diseases; host-parasite relationships; ecology and life history of parasites; epidemiology; therapeutics; new diagnostic tools.

All papers in Parasite are published in English. Manuscripts should have a broad interest and must not have been published or submitted elsewhere. No limit is imposed on the length of manuscripts.

Parasite (open-access) continues Parasite (print and online editions, 1994-2012) and Annales de Parasitologie Humaine et Comparée (1923-1993) and is the official journal of the Société Française de Parasitologie. 